\title{
LIMITATIONS AND FUTURE OF RETICON DETECTORS
}

Gordon A. H. Walker

Geophysics and Astronomy Department

University of British Columbia

Vancouver, B.C., V6T 1W5, Canada

ABSTRACT. Linear arrays of self-scanned silicon diodes have been used in astronomical spectroscopy for over a decade. With care in the flat-fielding and data reduction they can be calibrated to better than $0.1 \%$. They are still the best detector for signal to noise levels $>100$ when continuous wide-band coverage is needed. CCD's should be capable of this spectrophotometric performance but, for the forseeable future, the lack of a large format and their high cost only make them competitive for spectroscopy of single spectral features or multiple echelle spectra.

\section{INTRODUCTION}

"It is quite apparent that from now on the photographic plate will be considered a specialised detector. Arrangements for its use will have to be made well in advance much the same as was done in the past for the electronic detectors." J.M. Fletcher DAO, January 7. 1987 on $f$ inding that no plates were taken with the $1.8 \mathrm{~m}$ telescope in 1986 .

While the Reticon is unlikely to have as long a reign as the photographic plate, it is clear from the wealth of results being presented at this meeting that it will remain part of the equipment at most observatories for at least the rest of this century. At CFHT it is used for one third of the observations.

Self-scanned, linear-arrays of silicon diodes were first introduced by the Reticon corporation (now E. G. and G. Reticon) in 1971 for optical character recognition, facsimile production, and non-contact control. The first devices had 64 diodes and they are now available with up to 4096. Tens of thousands are produced each year, mostly for optical character recognition at check-out counters. The major scientific use is in multichannel spectrometers for chemistry with astronomy only taking a tiny fraction of the 
total production. Unlike the situation with CCD's, astronomers seem assured of a secure supply of quality diode arrays at a reasonable price (a selected device costs only a few thousand $\$$ ).

Their geometrical stability, high panchromatic responsive quantum efficiency, high storage capacity, and almost total lack of lag make diode arrays superior to other signal generating detectors for high signal to noise spectroscopy. Tull and Nather (1973) were the first to report the use of a refrigerated diode array for astronomical spectroscopy and they are now in routine use for analog integration at most major and many smaller observatories. I counted over 200 papers based on their use when preparing this review. Reticons are widely used in astronomy in other than an integrating mode e.g. photon-counting detecting phosphor scintillations or electron bombarded (Digicon), and in radio-frequency acousto-optic spectrometers. (E. G. and G. Reticon are planning to build a $512 \times 512$ CCD suitable for astronomy.)

\section{RETICON vS. CCD}

Reticons have a highly linear response because each photon detected produces a single charge-carrier pair but the lack of internal gain introduces a large read-out noise. Detective Quantum Efficiency, DQE, measures the efficiency with which a detector reproduces the $N^{0} \cdot 5$ signal to noise in an incident beam of $\mathrm{N}$ photons. For a single diode:

$\mathrm{DQE}=\mathrm{Q} /\left(1+(\text { read-out noise })^{2} / \mathrm{NQ}\right)=((\mathrm{S} / \mathrm{N} \text { out }) /(\mathrm{S} / \mathrm{N} \text { in }))^{2}$, where $Q$ is the responsive quantum efficiency, and the (read-out noise) ${ }^{2}$ is measured in equivalent detected photons. Q for the Reticon is almost optimal for a silicon device as can be seen in Figure 1 where $Q$ is shown as a function of wavelength for several front and rear illuminated $C C D^{\prime} s$, a semi-transparent S-20 photocathode, and the RL 1872 Reticon. The dashed line indicates $Q$ enhancement using a fluorescent coating on the thick CCD's (Cullum, Deires, D'Ordico, and $\operatorname{Re} i \beta(1985))$.

The limiting read-out noise for both Reticons and CCD's is KTC switching noise $\left(=2.5 \times 10^{9}\right.$ (KTC) $0^{\cdot 5}$ electrons). For diode arrays most of the capacitance is in the diffusion associated with the shift registers, while for the CCD it is largely the input node capacitance of the on-chip amplifier. For the Reticon $C$ is of the order of 1 pF while for a CCD it is only about $1 \mathrm{fF}$ ( $i . e$. about $10^{-3}$ less).

Figure 2 shows the improvements which we have made in the read-out noise of our RL $1872 \mathrm{~F} / 30$ systems (Walker, Johnson and Yang (1985)) based on the pioneering work of Geary (1979), Livingstone, Harvey, Slaughter, and Trumbo $(1976)$, and Vogt $(1981)$. With the three systems we have built for CFHT, DAO, and UBC we regularly achieve read-out noise values of less than 400 electrons rms which is close to the expected (KTC) ${ }^{0.5}$ limit. In the following discussion 


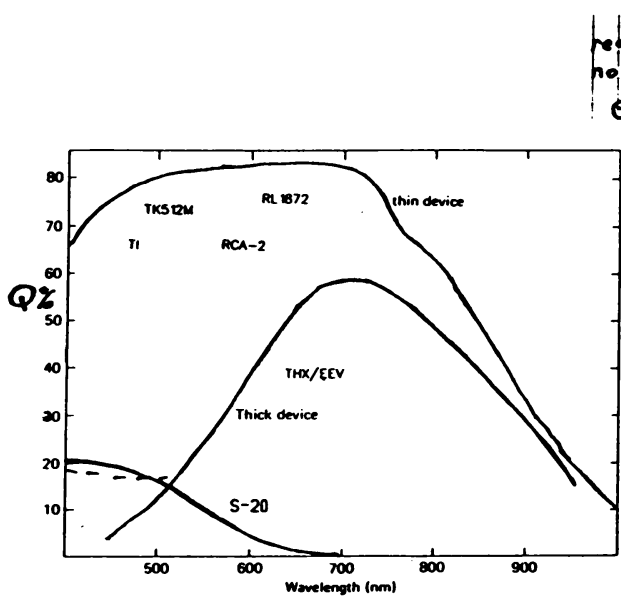

FIGURE 1. Variation of Responsive Quantu Efficiency For various silicon devices and seai-transparent

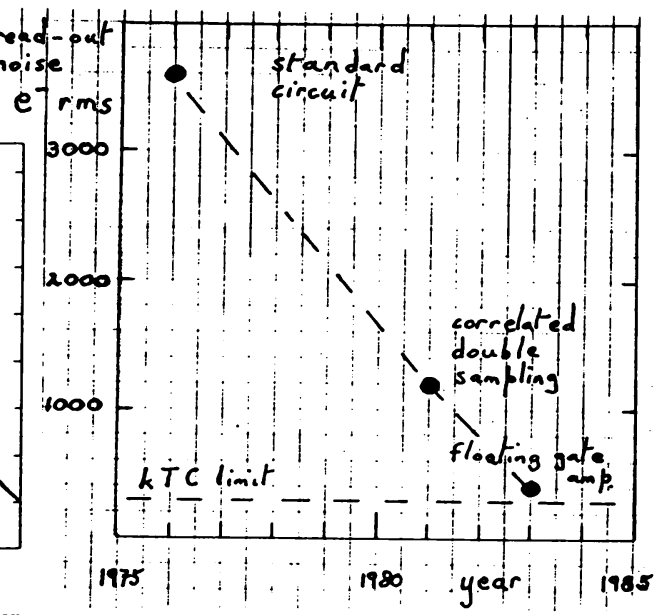
S-20 photocathode.

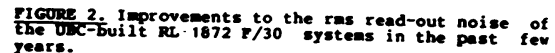
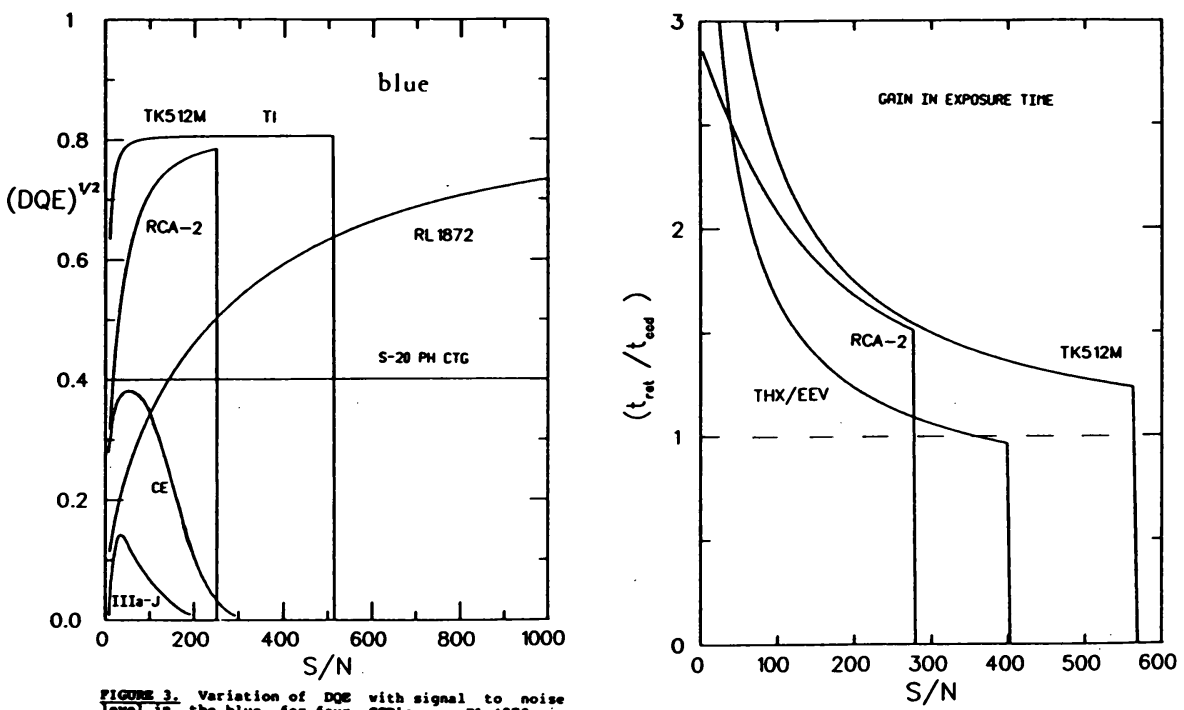

plove 3. Variation of Doe vith signal to noise

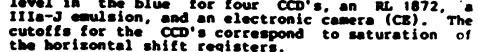

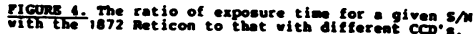


I adopt the very conservative value of 500 electrons rms.

Figure 3 shows how (DQE) $0^{\cdot 5}$ varies with detected signal

to noise for various detectors in the blue. Binning is assumed for the CCD's and the cut-off at high $S / N$ corresponds to saturation of the horizontal shift register. The curve for the IIIa-J emulsion is for an area of 1000 square microns and was taken from Hoag (1976) while the curve for the Electronic Camera is an educated guess, again for 1000 square microns. It is clear that within their range of operation the silicon devices are markedly superior, with the CCD's being even better than the Reticon.

of more importance to the astronomer than these technicalities is just what is the difference in exposure times implied by Figure 3. This is illustrated in Figure 4 where the the exposure time required with the Reticon is compared with that needed with the four different CCD's to achieve a given signal to noise ratio. In all cases the exposure is longer for the Reticon and catastrophically so for $S / N<100$. However, this does not take account of the simultaneity gain of the Reticon which has more pixels in-line than the CCD's. This is remedied in Figure 5 where the curves of Figure 4 have been multiplied by the ratio of the number of pixels in each device. An 1872 Reticon is assumed. In this case the Reticon is superior above $\mathrm{S} / \mathrm{N}=$ 50. This advantage is ofcourse regained by CCD's when used in an echelle spectrograph but, by the same token, some groups are implementing the 4096 Reticon array and at the University of Texas Tull and Young now have the 'Octicon', eight 1872 Reticons, operating successfully, to give $1.5 \times 10^{4}$ diodes in line! It is worth remembering that the proposed $2048 \times 2048$ Tek CCD will cost >\$105!

The above analysis suggests that for:

(i) $S / N<250$, use a CCD for single spectral features or multiple echelle spectra,

(ii) $\mathbf{S} / \mathbf{N}>100$, use a Réticon for wide continuous spectral coverage. (For $\mathbf{S} / \mathrm{N}<100$, unless exposure times are short, spectra become progressively contaminated by cosmic ray muon events see for e.g. Walker $(1987))$.

(iii) $S / N>250$, only use a Reticon unless the reported photometric calibration of CCD's can be improved by an order of magnitude from the current $1 \%$ level.

\section{CALIBRATION}

I am confident that CCD calibrations will eventually improve although it would be interesting to know what limitations are introduced by scattered light and other problems in echelle spectrographs. It is certainly possible with care to calibrate Reticons to better than $0.1 \%$.

As many groups seem to have difficulty achieving the high photometric performance of which the Reticon is capable I briefly outline below our flat fielding procedures. 


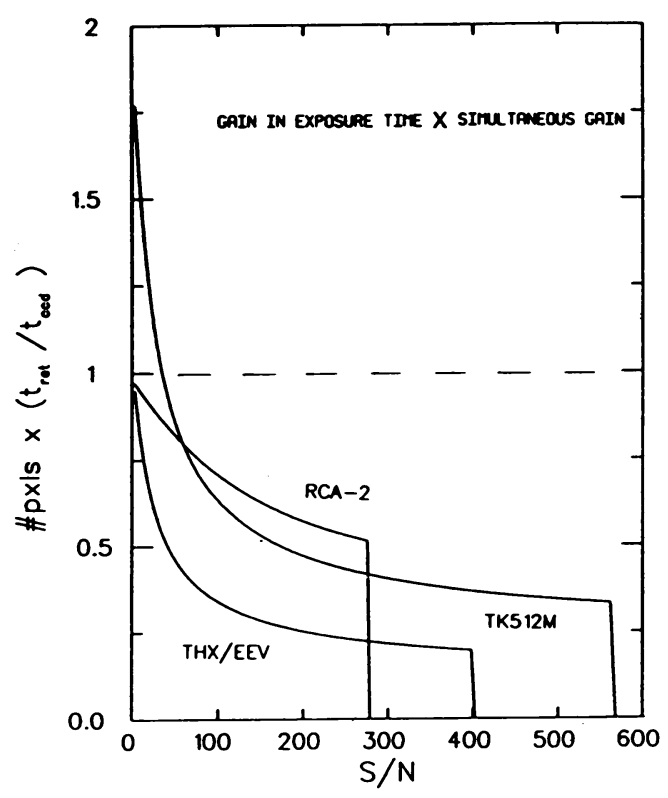
PIcops 5. The save as rig. Sut for the sape number
of spectral resolut ion elements for each detector.

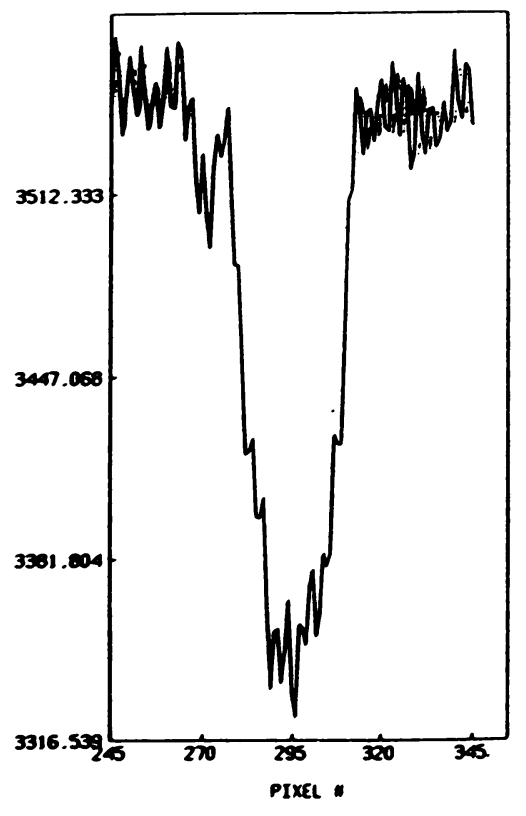

ripose 7,100 diode portion of the spoctry of spice

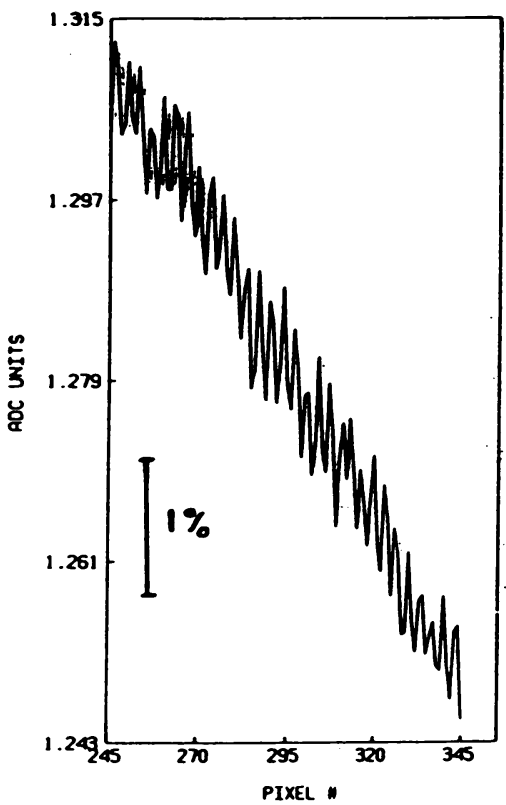

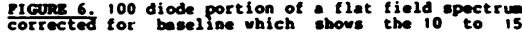
quasi-periodic wariation of 0 with diode number.

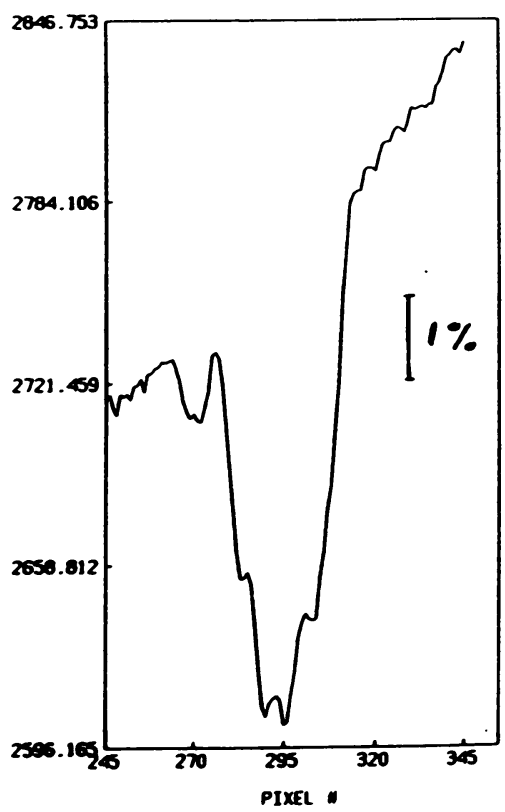

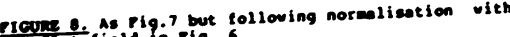
Gicuse o As rig.7 but 6. 
Diode to diode variations in $Q$ show a quasi-periodicity of ten to fifteen diodes at a level of about half of one percent. This can be very clearly seen in Figure 6 which shows a 100 diode portion of a flat field spectrum after subtraction of the baseline. Unless star and flat-field light fill exactly the same area of the array the quasi-periodic bumps will appear at some level in the final spectra where they can be mistaken for real features as well as confusing radial velocity measurements based on small shift techniques.

Spectra at CFHT, DAO, and UBC are taken with a Richardson image slicer (Richardson, Brealey, and Dancey 1971) which focusses a one dimensional image of the telescope exit pupil on the detector, normal to the direction of dispersion. Each of the coudé spectrographs is fed by a small-mirror coudé train which operates at a large intermediate $\mathrm{f} / \mathrm{ratio}$ with a focal reducer before the slicer (Richardson et al. 1971). The reducing lens provides an accessible exit pupil between lens and slicer which can be located by eye.

To eliminate residual collimation errors, the exit pupil is isolated by adjusting the position of an iris diaphragm (with a central disc to cover the secondary mirror shadow) for each star. It is sometimes even necessary to move it during a long spectral series. The iris is illuminated by a lamp and diffuser to give the flat field. The array is aligned 'vertically' on the spectrum of the flat field.

Figures 7 and 8 show 100 diodes of the spectrum of Spica unnormalised and normalised by the flat-field shown in Figure 6, respectively. It is clear from Figure 8 that the calibration is good to better than the 0.18 level. For complete processing the spectrum should be filtered to $50 \%$ of the Nyquist frequency if the line spread function has been properly sampled by the diode spacing.

\section{REFERENCES}

Cullum, M., Deires, S, D'Ordico, S., Reiß, R., 1985, Astron. Astrphys., 153, L1.

Geary, J. C., 1979, Proc. S.P.I.E., 172, 82.

Hoag, A.A., 1976, AAS Phot. Bull., 13, 14.

Livingstone, W.C., Harvey, J., slaughter, C., Trumbo, D., 1976, Appl. Opt, 15, 40 .

Richardson, E.H., Brealey, G.A., Dancey, R., 1971, Publ. Dom. Ap. Obs., 14, 1.

Tull, R.D., Nather, R.E., 1973, Astr. Obs. with TV Sensors, UBC, ed. Glaspey and Walker, 171.

vogt, S.S., 1981, Proc.S.P.I.E., 290, $70^{\circ}$

Walker, G.A.H., Johnson, R., Yang, S., 1985, Adv. Electronics El ectron Phys., 64A, 213.

Walker, G.A.H., 1987, Astronomical Observations, Cambridge University Press. 